\section{A FORTRAN program for Cohen's kappa coefficient of observer agreement}

\author{
RONALD A. BERK \\ The Johns Hopkins University, 5485-S Harper's Farm Road \\ Columbia, Maryland 21044 \\ and \\ KENNETH L. CAMPBELL \\ Montgomery County Public Schools, 850 Hungerford Drive \\ Rockville, Maryland 20850
}

The agreement or consistency of independent observations recorded by two raters has been computed using a variety of procedures. They range from the simplest, most inadequate method of the raw percentage/proportion of cases in which both raters agree to the most precise, comprehensive approach of the intraclass correlation which involves the analysis of variance components (Ebel, 1951). Among the proposed methods between these extremes is a measure of observer agreement for nominal scales developed by Cohen (1960), the " $K$ " coefficient. It is directly interpretable as the proportion of observer agreement that exists over and above that which would occur by chance.

The " $k$ " coefficient is based on the cell and marginal frequencies of the observation categories in a two-dimensional (C by $\mathrm{C}$ ) contingency table. An exemplary table for two observers and three categories is shown below:

$\begin{array}{lccccc} & & & \text { Observer 1 } & \text { Row } \\ & 1 & n_{11} & n_{12} & n_{13} & n_{1+} \\ \text { Observer 2 } & 2 & n_{21} & n_{22} & n_{23} & n_{2+} \\ & 3 & n_{31} & n_{32} & n_{33} & n_{3+} \\ \text { Column Total } & & n_{+1} & n_{+2} & n_{+3} & N\end{array}$

The computational formula is:

$$
k=\frac{p_{o}-p_{e}}{1-p_{e}},
$$

where $p_{0}$ equals:

$$
\sum_{i=1}^{c} n_{i i} / N,
$$

and $p_{e}$ equals:

$$
\sum_{i=1}^{C} n_{i+} n_{+i} / N^{2}
$$

The former represents the observed proportion of agreement, while the latter represents the proportion of agreement expected by chance. The coefficient essentially compares the observed entries in the main diagonal of the table with the expected entries on this diagonal, where the expected frequency is computed from the standard chi-square multiplicative model of independence (Light, 1973, p. 331).

The statistical significance of $\kappa$ is tested with the large-sample $(n \geqslant 100)$ normal approximation of the form:

$$
Z=\frac{\kappa}{\sqrt{\frac{P_{e}}{N\left(1-p_{e}\right)}}} .
$$

This tests the null hypothesis of random agreement.

The standard error of $k$ is expressed as:

$$
\sigma_{\kappa}=\sqrt{\frac{\mathrm{p}_{\mathrm{o}}\left(1-\mathrm{p}_{\mathrm{o}}\right)}{\mathrm{N}\left(1-\mathrm{p}_{\mathrm{e}}\right)^{2}}},
$$

where $p_{e}$ is assumed constant and $p_{o}$ is the population value. This estimate can be used to establish confidence limits for a given value of $\kappa$.

Input. One data card is minimally required to execute the program. This card contains an alphanumeric label for the contingency table, the number of data cards for the analysis, the number of rows/columns in the table, and up to 22 cell frequencies (F3.0 format) in a prespecified order across rows. Continuation cards are used for tables larger than 4 by 4 . Twenty observation categories is the maximum. This format is repeated for any number of successive tabular analyses within a single run.

Output. The printed output consists of the following: (1) a listing of the input contingency table with cell and marginal proportions, (2) the observed and expected proportions of agreement, (3) the $k$ coefficient, (4) the $Z$ value and significance level for the one-tailed test, (5) the standard error of $\kappa$.

Computer and language. Written in ANSI FORTRAN, the program is compatible with machines such as the IBM 360/70, UNIVAC 1100 series, and CDC 6000 series.

Availability. A program listing and user's manual may be obtained from either author at no cost.

\section{REFERENCES}

CohEN, J. A coefficient of agreement for nominal scales. Educational and Psychological Measurement, 1960, 20, 37-46.

Esel, R. L. Estimation of the reliability of ratings. Psychometrika, 1951, 16, 407-424.

LigFT, R. J. Issues in the analysis of qualitative data. In R. M. W. Travers (Ed.), Second handbook of research on teaching. Chicago: Rand McNally, 1973. 